\title{
A Calculation Method for the Micro-vibration Amplitude of an Aerostatic Thrust Bearing and the Influence of Bearing Parameters on the Vibration
}

\author{
Yuntang Li ${ }^{1, a}$, Xin Wan ${ }^{1, b}$, Xiaolu Li ${ }^{1, c}$, Weihong Sun ${ }^{1, d}$ and Zai Sun ${ }^{2, e}$ \\ ${ }^{1}$ College of Mechanical and Electrical Engineering, China Jiliang University, No. 258, Xueyuan \\ Street, High Education District, Hangzhou 310018, China \\ ${ }^{2}$ College of Mechanical and Electrical Engineering, China Jiliang University, No. 258, Xueyuan \\ Street, High Education District, Hangzhou 310018, China \\ âuntangli@cjlu.edu.cn, ${ }^{b} 465716244 @ q q . c o m,{ }^{9}|x| 2006 @ c j l u . e d u . c n,{ }^{d}$ Whsun@cjlu.edu.cn \\ esunzai@cjlu.edu.cn
}

\begin{abstract}
Keywords: Aerostatic thrust bearing; Micro-vibration; Large Eddy Simulation (LES); Macro-vibration; Calculation method

Abstract. The micro-vibration caused by the locally unstable gas flow field of an aerostatic thrust bearing does always occur even if an invariable load acts on the bearing, which is harmful to the accuracy of an ultra-precision measuring device or manufacturing equipment. However, most researchers forecasted the vibration amplitude qualitatively. In this paper, Large Eddy Simulation (LES) was used to calculate the time-variation flow field of an aerostatic thrust bearing, and bearing force, stiffness and the fluctuant gas pressure on bearing surface were obtained. Further, a micro-vibration model was established and a method was proposed for calculation of the vibration amplitude. Experiments showed that the vibration could be forecasted quantificationally. In addition, the influence of bearing parameters on the vibration amplitude was analyzed numerically. The results indicated that the micro-vibration could be restrained if the bearing was featured with a large orifice diameter, a large bearing diameter, a shallow air chamber or a small air chamber diameter.
\end{abstract}

\section{Introduction}

An aerostatic thrust bearing with pressurized gas as lubricant has the merits of high precision, low wear, non-pollution and long life [1]. It is widely used in three-coordinate measuring machines, medical devices and microelectronic equipment. However, complicated vortices and even negative pressure (relative to bearing outlet) may exist inside the bearing as the flow field changes quickly after the gas effuses from orifice and shoots to bearing surface. The vortices or negative pressure causes pressure fluctuation and induces micro-vibration (in amplitude of sub-micrometers) of bearing body. Unlike the vibration caused by external disturbing force (macro-vibration in amplitude of micrometers), the micro-vibration does always occur even if an invariable load acts on the bearing. It is harmful to the accuracy of an ultra-precision measuring device or manufacturing equipment [2-3]. Moreover, the method for restraining macro-vibration can not reduce the micro-vibration because of different generation mechanisms. Although many researchers analyzed the mechanism and studied the methods for restraining the micro-vibration [4-6], they investigated the vibration based on the flow field at a transient time or the average pressure distribution inside the bearing.

Among the performance calculation methods for an aerostatic thrust bearing, Finite Difference Method (FDM), Finite Element Method (FEM) and Differential Quadrature Method (DQM) are widely used to analyze the pressure distribution inside the bearing. However, the Navier-Stokes equation is simplified to be Reynolds Equation and the feature structures of the bearing are omitted by FDM, FEM and DOM [7-9]. Reynolds Average Navier-Stockes (RANS) treats the turbulent flow as the superposition of average flow and transient pulsation. It can be used with approximations based on knowledge of the properties of flow turbulence to give approximate averaged solutions to the Navier-Stokes equations. Some researchers analyzed the performance of an aerostatic thrust being with RANS $[10,11]$. Direct Numerical Simulation (DNS) solves the Navier-Strokes equation without any turbulence model. It is suitable to solve the turbulent flow with low Reynolds number $(\mathrm{Re})$ 
instead of that with high Re because of high space and time resolutions due to the existence of multi-scale irregular flow. Large Eddy Simulation (LES) solves the turbulent motion larger than a certain scale. Unlike RANS, LES can capture large-scale effect and coherent structure existing in unsteady and unbalancing process. Compared with DNS, it can save calculation time as it does not need to solve the turbulent flow of all scales. Moreover, LES can analyze the flow status accurately and the calculation results coincide with experiments. Therefore, it is used to analyze the time-variation flow field inside an aerostatic thrust bearing in recent years [12].

In this paper, LES was used to calculate the time-variation flow field inside an aerostatic thrust bearing with a pocketed orifice-type restrictor and the results were verified by existing experiments and other calculation methods. Then, a micro-vibration model of the bearing was established based on the variables of bearing force, stiffness, gas film damping coefficient and the fluctuant pressure on bearing surface. With this model, the vibration amplitude was calculated quantificationally. Moreover, validity of the calculation results was evaluated by experiments and the deviations between numerical results and experiments were discussed. Finally, the influence of bearing parameters on the vibration amplitude was analyzed numerically so that it could be helpful for designing an aerostatic thrust bearing with low micro-vibration.

This paper is organized as follows: the next section verifies the results of LES by existing experiments and other numerical methods. Subsequent section proposes a calculation method for the micro-vibration amplitude of an aerostatic thrust bearing, and discusses the deviations between numerical results and experiments. Later section investigates the influence of bearing parameters on the vibration amplitude numerically. Conclusions are given in the last section.

\section{Verification of LES Results}

An aerostatic thrust bearing with a pocketed orifice-type restrictor consists of bearing body, lubricating gas and bearing surface, as shown in Figure 1. Where $d$ is orifice diameter, $D_{1}$ is air chamber diameter, $D$ is bearing diameter, $h$ is film thickness, $h_{1}$ is air chamber depth and $l$ is orifice length. The pressure distribution on bearing surface determines the static and dynamic performance of the bearing. In order to predict the micro-vibration amplitude, the time-variation pressure distribution on bearing surface was calculated by using LES. The theory, calculation model and setup of LES were illustrated in previous work [13]. In the calculation, 2D model was adopted as the calculation results can be obtained by defining the bearing was an axisymmetric structure. However, the 2D model can save calculation time and its calculation results are close to those of 3D model. Moreover, the effect of film thickness variation induced by the micro-vibration on LES results was neglected. Therefore, the mesh was invariable when the time-variation flow field was calculated at given film thickness.

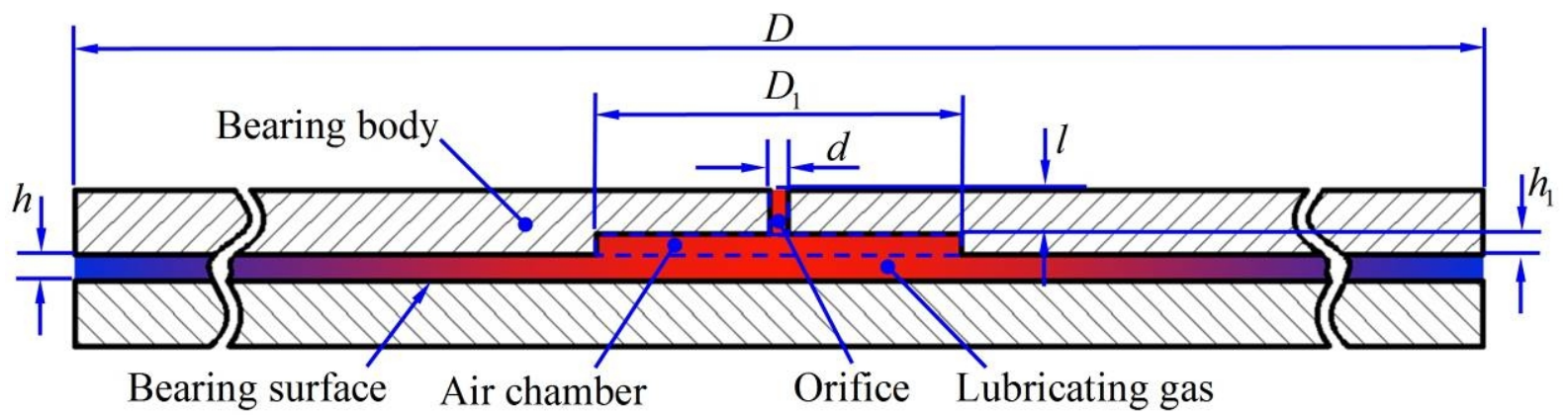

Fig. 1 Aerostatic Thrust Bearing with a Pocketed Orifice-type Restrictor

The calculation results of work [13] demonstrated that the pressure on bearing surface fluctuated rapidly. However, it is difficult to evaluate the LES results by measuring the fluctuant pressure and vortex intensity directly because the film thickness is only $4-20 \mu \mathrm{m}$ and time step is $10^{-5} \mathrm{~s}$. Therefore, the fluctuant pressure and vortex intensity is not measured directly in this paper. The average pressure on bearing surface calculated by LES was compared with those by the Navier-Stokes equation (N-S Eq), 3-region method (3-Region), Reynolds equation (Reynolds Eq) and experiments. The bearing 
parameters, numerical results of N-S Eq, 3-region, Reynolds Eq and experiments were given in Reference [14]. Figure 2 indicates that the results of different calculation methods agree with experiments if the flow field is away from orifice $\left(P_{\mathrm{s}}\right.$ and $\mathrm{R}$ are dimensionless supply pressure and dimensionless radius coordinate, respectively. $R_{\mathrm{s}}$ is the ratio of orifice radius to bearing radius). However, the deviations between numerical calculations and experiments become large when $\mathrm{R}$ is less than 0.2. The figure demonstrates that LES can accurately forecast the pressure distribution inside an aerostatic thrust bearing. Moreover, the validation of LES results was also verified in reference [12].

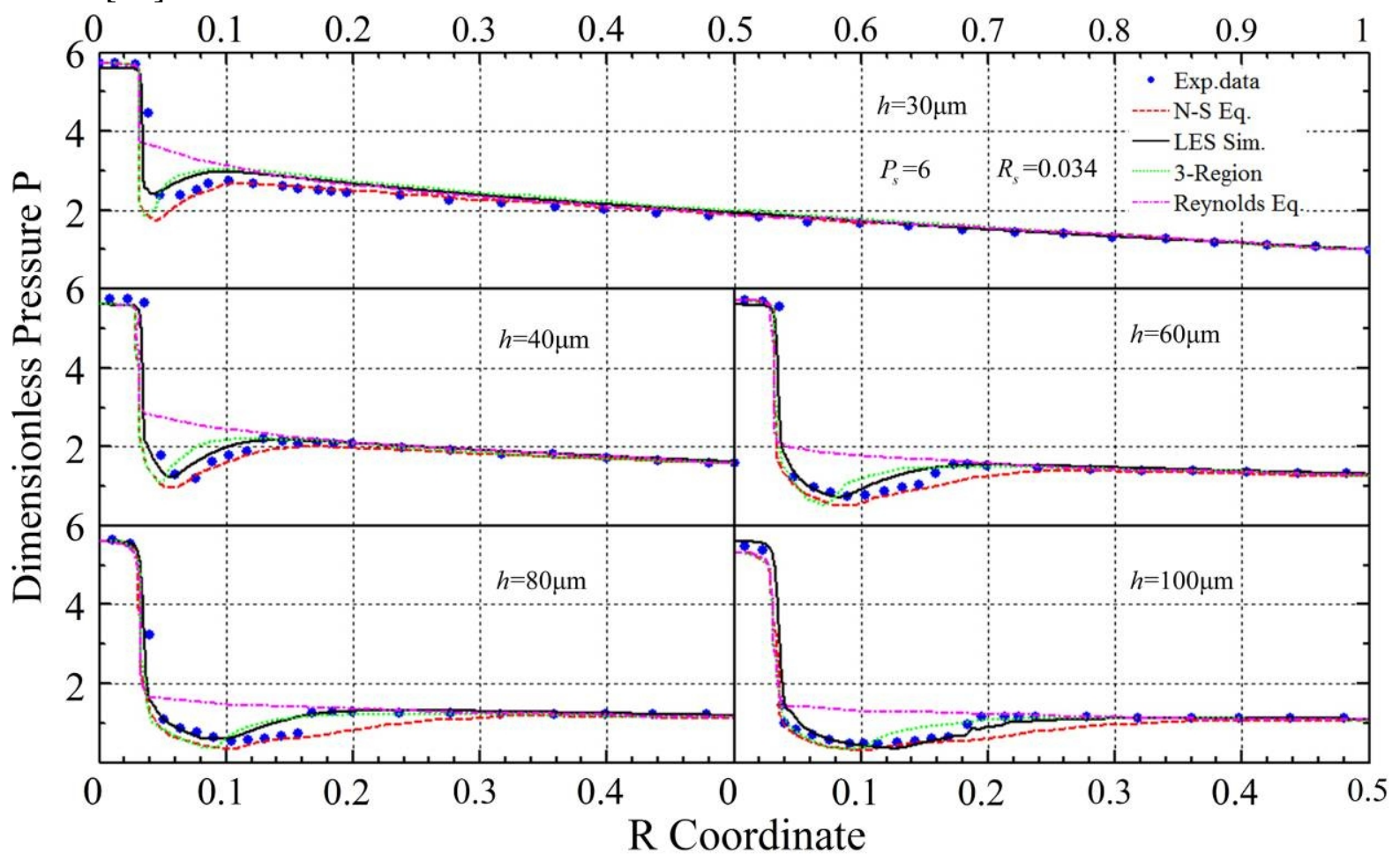

Fig. 2 Results of LES, Experiments and Other Calculation Methods

\section{Calculation Method for the Micro-vibration Amplitude of an Aerostatic Thrust Bearing}

\section{Micro-vibration Model of an Aerostatic Thrust Bearing}

Usually, researchers forecast qualitatively the micro-vibration of an aerostatic thrust bearing based on transient pressure or average pressure on bearing surface. By analyzing the influence of geometrical parameters, supply pressure and film thickness on the pressure distribution, the restraining method for the vibration is investigated. However, quantitative calculation the vibration amplitude is seldom carried out. The reason is that it is difficult to get the time-variation pressure distribution on bearing surface for establishment of the micro-vibration model. By using LES, the fluctuant pressure on bearing surface can be obtained and the vibration model can be established as follows.

An aerostatic thrust bearing is assumed as a mass spring-damper system, as shown in Figure 3. $f_{0}$ and $\Delta f(t)$ are static bearing force generated by average pressure $p_{0}$ and fluctuant bearing force induced by the fluctuant pressure $\Delta p$ (difference between transient area-weighted average pressure and $p_{0}$ ), respectively. $m$ is the mass supported by $f_{0}, k$ is stiffness, $\xi$ is gas film damping coefficient and its value is 0.04-0.06 according to the report of Reference [15]. $h_{0}$ and $\Delta h(t)$ are film thickness and micro-vibration amplitude, respectively. The bearing force at the $i$ th time step is

$$
F\left(t_{i}\right)=f_{0}+\Delta f\left(t_{i}\right)=\frac{1}{4} \pi D^{2}\left[p_{0}+\Delta p\left(t_{i}\right)\right]
$$

$\Delta p\left(t_{i}\right)$ is the fluctuant pressure at the $i$ th time step. $m$ is calculated by 
where $\mathbf{g}$ is $9.81 \mathrm{~m} / \mathrm{s}^{2}$. Supposing bearing force is constant during $t_{i}<t \leq t_{i+1}$ as time step is small enough $\left(1 \times 10^{-5} \mathrm{~s}\right)$, the micro-vibration model of the bearing is written as Eq. (3) if no external disturbing force acts on the bearing.

$$
\begin{aligned}
& m(t)+\xi h(t)+k h(t)=F\left(t_{i}\right) \\
& h(t)=h_{0}+\Delta h(t)
\end{aligned}
$$

Stiffness $k$ is calculated by using Eq. (5)

$$
k=\Delta f_{0} / \Delta h_{0}
$$

Eq. (6) can be obtained by substituting Eq. (4) into Eq. (3) considering $h_{0}$ and $f_{0}$ are constant.

$$
m \Delta(t)+\xi \Delta h(t)+k \Delta h(t)=\left.\Delta f\left(t_{i}\right) \quad \frac{}{h_{0}+\Delta h(t) \uparrow}\right|^{f_{0}+\Delta f(t)}
$$

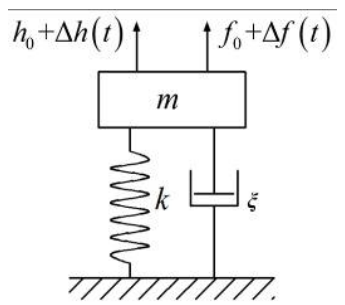

Fig. 3 Spring-damping System

Supposing the initial conditions were as follows: micro-vibration amplitude $\Delta h\left(t_{1}\right)=0$ and vibration velocity $\Delta h\left(f_{1}\right)=0$, the mass vibrates due to the excitation of $\Delta f\left(t_{1}\right) . \Delta h(t)$ and $\left.\Delta h \mathcal{F}_{t}\right)$ can be obtained when $t_{1}<t \leq t_{2}$ by resolving Eq. (6). Next, substituting $\left.\Delta h\left(t_{2}\right), \Delta h f_{t_{2}}\right)=0$ and $\Delta f\left(t_{2}\right)$ into Eq. (6), and then $\Delta h(t)$ and $\left.\Delta h f^{(} t\right)$ can be calculated when $t_{2}<t \leq t_{3}$. In the same way, one can get the vibration amplitude $\Delta h\left(t_{i}\right)$ at the $i$ th time step.

Figure 4(a) is the fluctuant pressure $\Delta p\left(t_{i}\right)$ on bearing surface of an aerostatic bearing with a pocketed orifice-type restrictor $\left(D=40 \mathrm{~mm}, d=0.1 \mathrm{~mm}, D_{1}=6 \mathrm{~mm}, h_{1}=0.1 \mathrm{~mm}, P_{s}=0.5 \mathrm{MPa}, h_{0}=12 \mu \mathrm{m}\right.$, $l=0.5 \mathrm{~mm})$. The calculated micro-vibration amplitude is shown in figure $4(\mathrm{~b})$ when the gas film damping coefficient $\xi$ is assumed as 0.05. Figure 4(c) is the Power Spectrum Density (PSD) of the vibration amplitude. It is concluded that the proposed method can forecast the vibration quantificationally and the maximum vibration amplitude is $43.3 \mathrm{~nm}$. Moreover, the vibration has a bandwidth of several thousand hertz. Figure 4(d) is the fluctuant pressure when film thickness is $12.05 \mu \mathrm{m}$. The maximum fluctuant pressure decreases from $384 \mathrm{~Pa}$ to $372 \mathrm{~Pa}$ compared with that when film thickness is $12 \mu \mathrm{m}$. Therefore, the effect of film thickness variation on LES results can be neglected. 


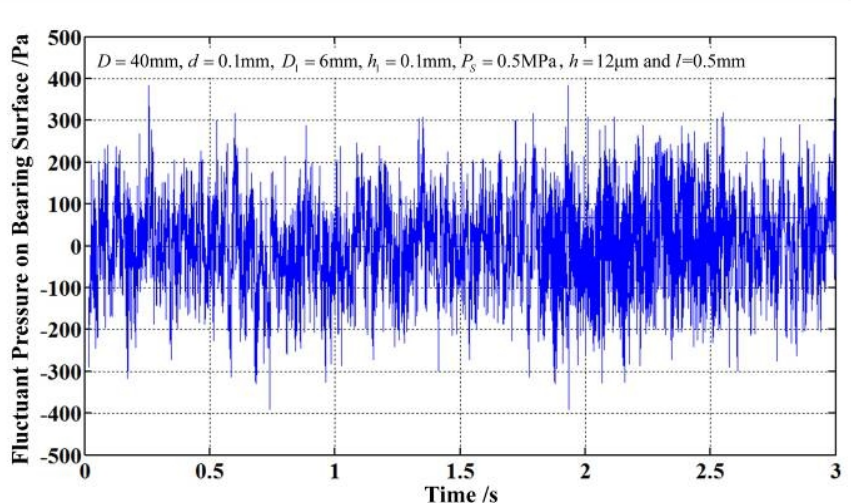

(a) Fluctuant Pressure on Bearing Surface when $h$ is $12 \mu \mathrm{m}$

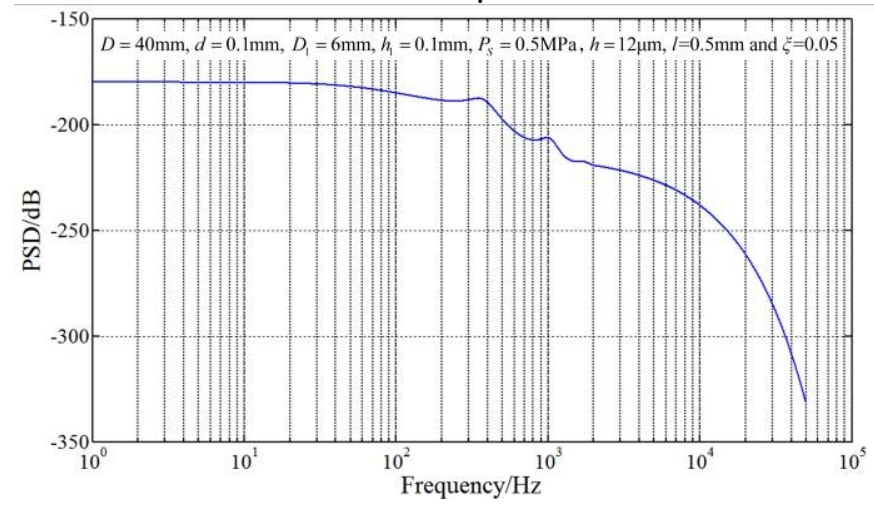

(c) Power Spectrum Density of the Micro-vibration Amplitude

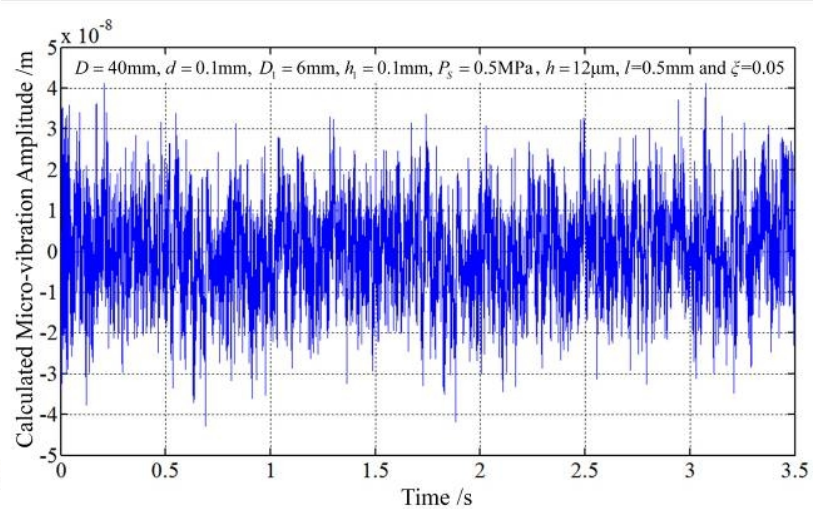

(b) Calculated Micro-vibration Amplitude

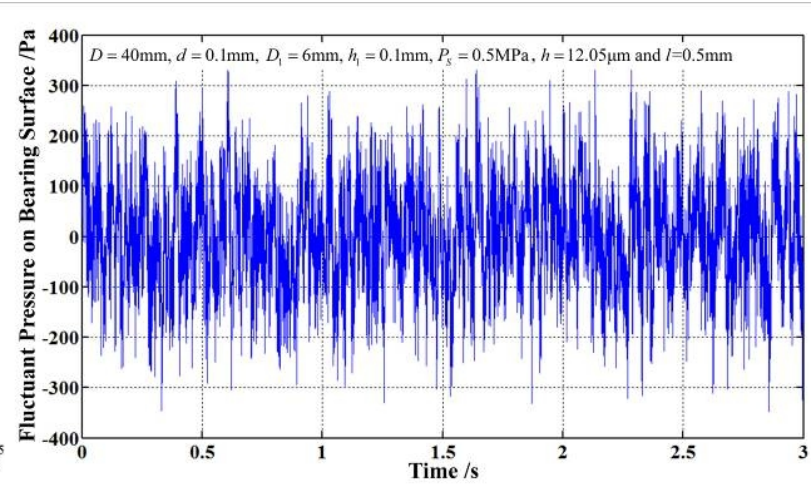

(d) Fluctuant Pressure on Bearing Surface when $h$ is $12.05 \mu \mathrm{m}$

Fig. 4 Micro-vibration Amplitude Calculation

\section{Experiments}

Experiments were carried out on a device for performance-testing of an aerostatic thrust bearing, as shown in Figure 5(a). The device consisted of two drive motors, four adjusting bolts, a magnetic meter base, a displacement sensor (Type GT2-H12K, made by Keyence Corporation, measurement range: $12 \mathrm{~mm}$, resolution: $0.1 \mu \mathrm{m}$ ), a force transducer (measurement range: $100 \mathrm{Kg}$, resolution: $0.1 \mathrm{Kg}$ ), a lever, a mass, a ball screw, a connecting rod, a ball club, a marble plate, a motion plate and a marble base.

The experiments were conducted as follows:

Step 1: Measurement of Load Carrying Capacity (LCC) with different film thicknesses.

Step 2: Micro-vibration testing. It is difficult to gauge the micro-vibration amplitude directly as it is on a sub-micrometer scale and the vibration frequency ranges from several hertz to several thousand hertz. Therefore, the acceleration of the vibration was tested and it was transferred to frequency domain by using Fast Fourier Transform (FFT). The vibration amplitude was obtained by double integral of the acceleration signal in frequency domain before the signal was transferred to time domain again by using Inverse Fast Fourier Transform (IFFT).

In the acceleration measurement, a load equal to LCC (obtained in Step 1) generated by a set of weights was fastened to the bearing. Three acceleration sensors (Type 352C42 made by PCB Corporation, $120^{\circ}$ symmetrically disposed) were used to test the micro-vibration. Signals of the acceleration sensors were collected by a vibration noise analyzer (CoCo-80, made by Crystal Instruments Corporation). Moreover, the vibration of the marble plate was measured by an acceleration sensor (Type 4507, made by B\&K Corporation) in order to monitor the environmental vibration.

Figure 6 shows that the maximum vibration amplitude is $53.9 \mathrm{~nm}$ when an aerostatic bearing has the same parameters as those given in Section 3.1. The reason for the disagreement of numerical 
calculation curve with experiments is that the fluctuant pressure generated by the flow field inside the bearing is complicated and changes fast. Moreover, the calculation time can not be synchronous to that of experiments. However, the predicting method is of importance because quantitative calculation the micro-vibration amplitude is helpful for evaluation of the vibration restraining methods and research of the influence of bearing parameters on the vibration.

\section{Analysis of the Deviations between Numerical Results and Experiments}

The deviation of the maximum vibration amplitude between numerical calculations (see

Section 3.1) and experiments (see Section 3.2) is 17.6\%. We speculate the reasons are as follows and future work is needed to improve the calculation accuracy.

i) The bearing force on bearing surface within a time step was assumed as constant in the proposed micro-vibration amplitude calculation method. The calculation accuracy can be improved by decreasing the time step.

ii) The gas film damping coefficient was regarded as constant. However, it changes with film thickness. The calculation accuracy can be increased if nonlinear gas film damping coefficient is modeled in Eq. (3).

iii) The measurement accuracy of film thickness, vibration acceleration and bearing force affected the experimental results.

iv) The manufacturing accuracy of the bearing.

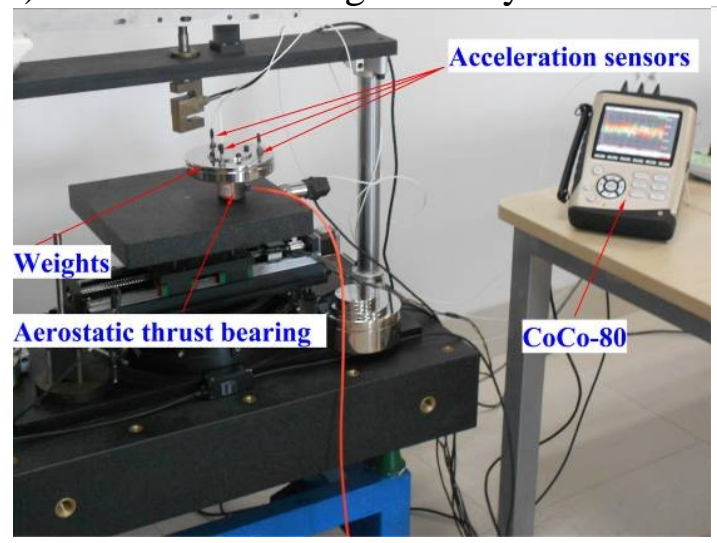

Fig. 5 Experiments

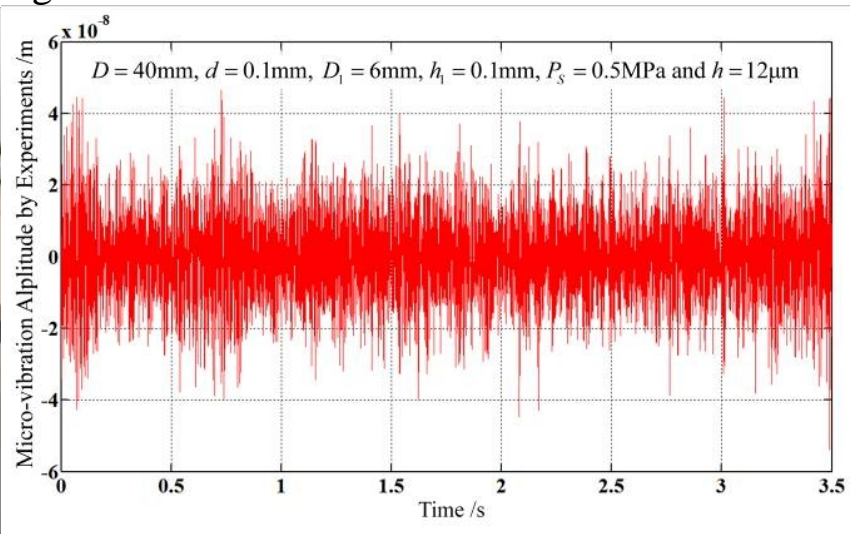

Fig. 6 Micro-vibration Amplitude Obtained Experimentally

\section{Influence of Bearing Parameters on Micro-vibration Amplitude}

The micro-vibration amplitude calculation method for an aerostatic thrust bearing provides a way of ascertaining quantificationally the influence of bearing parameters on the vibration. In the following section, the influence of the orifice diameter, bearing diameter, air chamber depth and air chamber diameter on the vibration amplitude is analyzed numerically.

In proposing the micro-vibration amplitude calculation method mentioned in Section 3.1, the gas film damping coefficient $\xi$ was not detected but regarded as constant. In order to make the following analysis reasonable, the influence of $\xi$ on the vibration amplitude was analyzed.

Figure 7 shows that the maximum vibration amplitudes with $\xi$ of $0.04,0.045,0.05,0.055$, and 0.06 are $43.9,43.6,43.3,43.0$ and $42.7 \mathrm{~nm}$, respectively. The bearing parameters are the same as those discussed in Section 3.1. It is concluded that the vibration amplitude decreases with the growth of $\xi$. The maximum vibration amplitude error induced by $\xi$ is $2.8 \%$. Therefore, $\xi$ is specified as 0.05 and its influence on the vibration amplitude is neglected in the following analysis. 


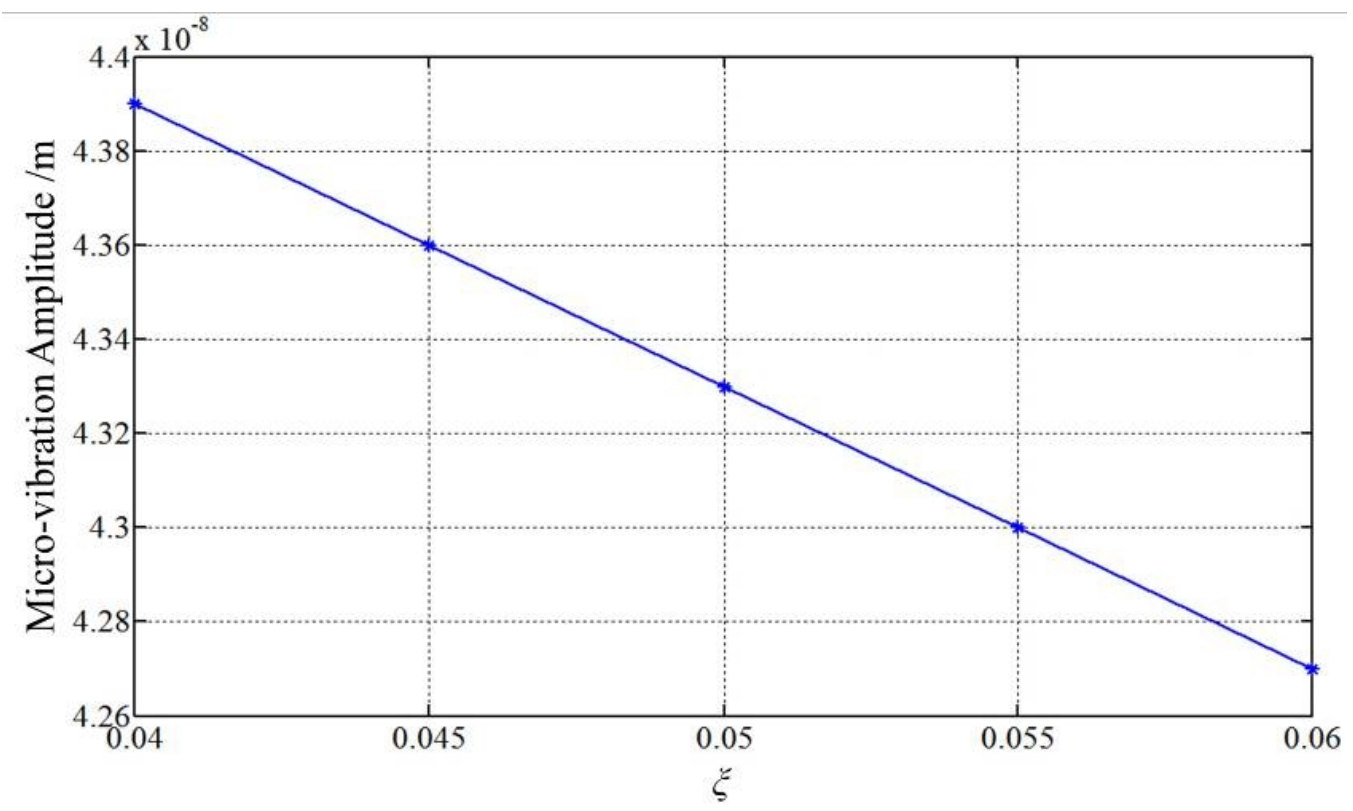

Fig. 7 Influence of Gas Film Damping Coefficient on Micro-vibration Amplitude

Figure 8 demonstrates the influence of orifice diameter, bearing diameter, air chamber depth and air chamber diameter on the maximum micro-vibration amplitude. The vibration amplitude is influenced by film thickness greatly. Moreover, it increases firstly with the growth of film thickness. Then, it begins to decrease with the increase of film thickness after it reaches the maximum value. The reason is that the fluctuant pressure is mainly induced by the locally unstable flow field in the air chamber near orifice outlet. The flow field becomes stable and the fluctuant pressure will reduce as flow resistance increases with the decrease of film thickness. Although the fluctuant pressure in air chamber increases with the growth of film thickness, it is far away from bearing surface when film thickness is large enough. Therefore, the vibration amplitude decreases with the growth of film thickness after it reaches the maximum value.

Figure 8(a) shows that the micro-vibration amplitude of the bearing with an orifice diameter of $0.1 \mathrm{~mm}$ is smaller compared with those with an orifice diameter of $0.15 \mathrm{~mm}$ and $0.2 \mathrm{~mm}$. The vibration amplitude increases with the growth of orifice diameter if other parameters are constant. For an aerostatic thrust bearing, the smaller the orifice diameter is, the larger the stiffness (the ability of the bearing maintaining stability when it is acted by an external disturbing force) is. Therefore, an aerostatic thrust bearing with a pocketed orifice-type restrictor will be stable under external disturbing force and the micro-vibration can be restrained as well when orifice diameter is small.

Figure 8(b) indicates that the micro-vibration amplitude of the bearing with a bearing diameter of $20 \mathrm{~mm}$ is larger compared with those with a bearing diameter of $30 \mathrm{~mm}$ and $40 \mathrm{~mm}$. The vibration amplitude decreases with the growth of bearing diameter if other parameters are constant. Generally the stiffness of an aerostatic thrust bearing increases with the growth of the bearing diameter. Therefore, the bearing with a large bearing diameter will be stable under external disturbing force and the micro-vibration can be reduced at the same time. 

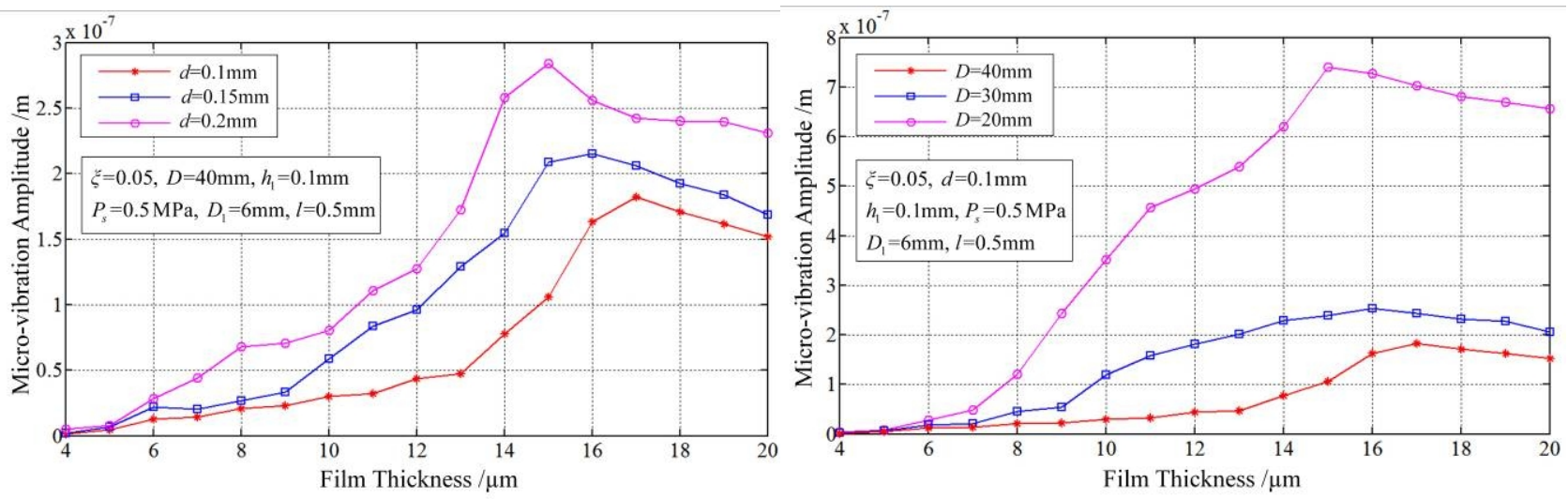

(a) Influence of $d$ on Micro-vibration Amplitude

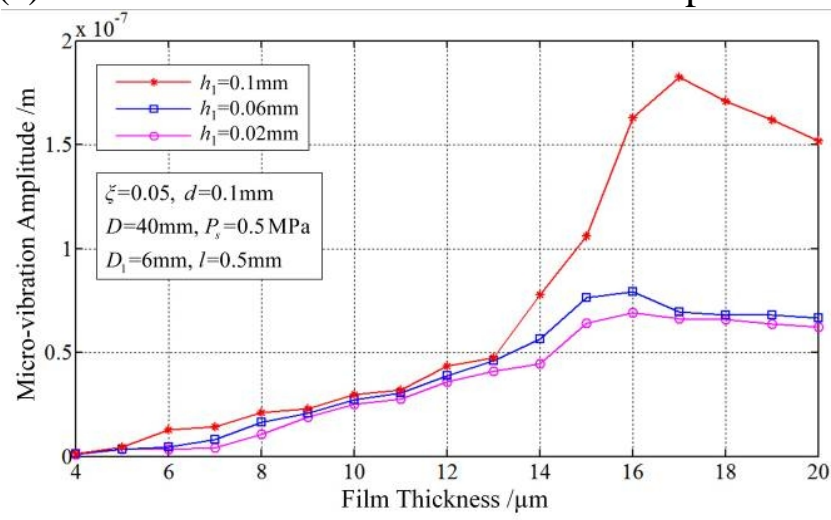

(b) Influence of $D$ on Micro-vibration Amplitude

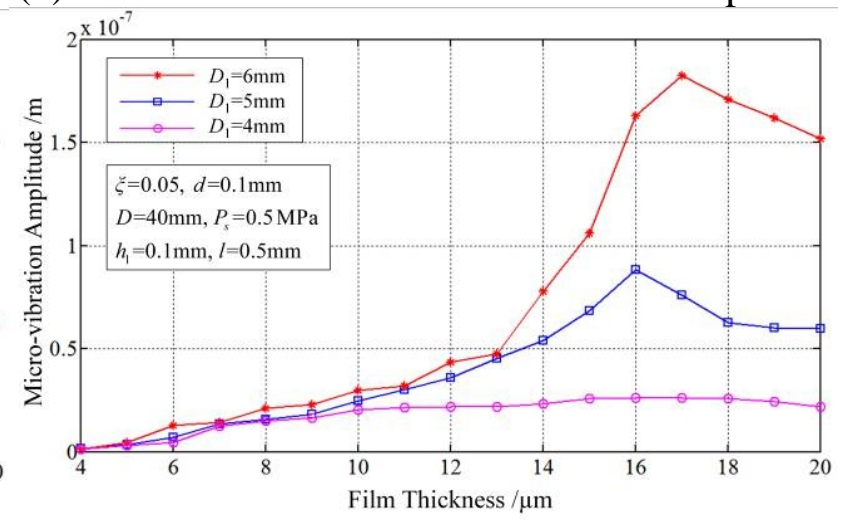

(c)Influence of $h_{1}$ on Micro-vibration Amplitude (d) Influence of $D_{1}$ on Micro-vibration Amplitude

Fig. 8 Influence of Bearing Parameters on Micro-vibration Amplitude

Figure 8(c) demonstrates that the micro-vibration amplitude of the bearing with an air chamber depth of $0.02 \mathrm{~mm}$ is smaller compared with those with an air chamber depth of $0.06 \mathrm{~mm}$ and $0.1 \mathrm{~mm}$. The vibration amplitude increases with the growth of air chamber depth if other parameters are constant. Although, a shallow air chamber depth is helpful for restraining the micro-vibration of an aerostatic thrust bearing, the pocketed orifice-type restrictor will become an inherent orifice-type one if air chamber is too shallow (the minimum area of the gas channel may be $\pi d\left(h+h_{1}\right)$ or $\pi D_{1} h$, not $\left.\pi d^{2} / 4\right)$. The stiffness of the bearing with an inherent orifice-type restrictor is better than that with a pocketed orifice-type one if other parameters are constant. However, the bearing with a pocketed orifice-type restrictor is widely used considering bearing force. Therefore, air chamber depth should be determined carefully when designing an aerostatic thrust bearing with low micro-vibration.

Figure 8(d) indicates that the micro-vibration amplitude of the bearing with an air chamber diameter of $4 \mathrm{~mm}$ is smaller compared with those with an air chamber diameter of $5 \mathrm{~mm}$ and $6 \mathrm{~mm}$. The vibration amplitude increases with the growth of air chamber diameter if other parameters are constant. The reason is that the area of the unstable flow field inside the bearing increases with air chamber diameter. The previous work about the performance of an aerostatic thrust bearing reported that a positive correlation exists between air chamber diameter and bearing force and stiffness [16]. Therefore, air chamber diameter should be selected carefully considering bearing force, stiffness and micro-vibration.

\section{Conclusions}

In this paper, a micro-vibration amplitude calculation method was proposed for an aerostatic thrust bearing with a pocketed orifice-type restrictor based on bearing force, stiffness, gas film damping coefficient and the fluctuant pressure on bearing surface. Experiments demonstrated that the method could forecast quantificationally the vibration amplitude, and it is helpful to evaluate a micro-vibration restraining method. Numerical calculations indicated that film thickness influenced 
the vibration amplitude clearly. Moreover, the vibration could be restrained if the bearing was featured with a large orifice diameter, a large bearing diameter, a shallow air chamber or a small air chamber diameter.

\section{Acknowledgements}

This study was sponsored by the National Nature \& Science Foundation of China under Grant 51275499 and 50905171, Nature \& Science Foundation of Zhejiang Province, China under Grant LY15E050014, and Research Project of State Key Laboratory of Mechanical System and Vibration (Shanghai Jiaotong University) under Grant MSV201516.

\section{References}

[1] M. E. Eleshaky. Tribol Int. Vol. 42(2009), p. 1108

[2] G. Aguirre, F. Al-Bender and V. B. Hendrik. Precis Eng. Vol. 34(2010), p. 507

[3] T. Kawai, K. Ebihara and K, Y. Takeuchi. CIRP Annals - Manufacturing Technology. Vol. 54(2005), p. 329

[4] X. D. Chen and X. M. He. 2006. Tribol Int. Vol. 39(2006), p. 1336

[5] X. D. Chen, H. Chen, X. Luo, Y. X. Ye, Y. T. Hu and J. Q. Xu. Tribol Let.t Vol. 42(2011), p. 179

[6] T. Aoyama, K. Koizumi K, Y. Kakinuma and Y. Kobayashi. CIRP Annals - Manufacturing Technology. Vol. 58(2009), p. 367

[7] W. J. Lin, J. P. Khatait, W. Lin and H. Z. Li. Conference on Control, Automation, Robotics and Vision. 2006, p. 1

[8] H. C. Yu and W. Q. Ma. Conference on Mechatronics and Automation. (2010) , p. 53

[9] Z. G. Dong, Y. Ding, P. K. Liu and H. Ding. P I Mech eng J-J Eng. Vol. 228(2014), p. 232

[10] Y. Xu and G. Q. Zhang. Procedia Engineering. Vol. 14(2011), p. 922

[11] T. C. Hsu, H. L. Chiang and C. Y. Lin. Ind Lubr Tribol. Vol. 58(2006), p. 269

[12] J. C. Zhu, H. Chen and X. D. Chen. J Fluid Struct. Vol. 40(2013), p. 42

[13] Y. T. Li, Y. X. Lin, H. X. Zhu and Z. Sun. Tribol T. Vol. 57(2014), p. 28

[14] S. Yoshimoto, M. Yamamoto and K. Toda. J Tribol-T ASME, Vol. 129(2007), p. 384

[15] C. H. Huang. Characteristics Analysis of the Virtual Hollow Rectangular Aerostatic Guide Way in Precision Linear Motion Stage. MS Thesis, National University of Science and Technology, Taiwan (2001)

[16] Y. T. Li and H. Ding.. Influences of The Geometrical Parameters of Aerostatic Thrust Bearing with Pocketed Orifice-type Restrictor on Its Performance. Tribol Int. Vol. 40(2007), p. 1220 